\title{
La pairémulation entre bénévolat activiste et professionnalisation ${ }^{1}$
}

\author{
Eve Gardien \\ MCF en Sociologie \\ Université Rennes2 \\ ESO (UMR 6590)
}

La pairémulation participe en France d'un ensemble plus vaste de "pairsaccompagnements " mis en œuvre par des personnes handicapées pour des personnes handicapées. La pairémulation est une pratique résolument par et pour des pairs ${ }^{2}$. La pairémulation s'inscrit dans une tradition de résistances au disablism ${ }^{3}$ importée des EtatsUnis. Son déroulement varie selon diverses modalités, formelles et informelles, bénévoles et rémunérées. Après une vingtaine d'années de développement en France, les activistes s'interrogent sur l'opportunité et la pertinence d'envisager une professionnalisation de cette pratique. Or, s'engager dans la voie de la professionnalisation suppose d'autres arrangements avec les secteurs sanitaire et médico-social, peut-être même de renoncer aux fondements de cette pratique, à savoir la résistance au disablism. Après une courte présentation de la pairémulation et de sa professionnalisation en cours, cet article visera à éclairer les différents points de débat entre pairémulateurs concernant la professionnalisation de cette pratique, ainsi que les argumentaires qui les sous-tendent.

\section{La Pairémulation : définition et pratiques}

Le vocable de "pairémulation » est né en France en 1994, lors des premières journées nationales pour la vie autonome 4 . La définition du terme " pairémulation » élaborée par les acteurs et activistes participants à ce rassemblement est : " la transmission de l'expérience par les personnes handicapées autonomes, pour les personnes handicapées en recherche de plus d'autonomie, avec le but de renforcer la conscience des personnes handicapées sur

\footnotetext{
${ }^{1}$ Soutenu par l'Agence Nationale de la Recherche (ANR), ce travail participe du programme SOCIORESIST. Ce projet scientifique propose d'interroger les différents dispositifs contemporains de la résistance ordinaire. Au nombre de ceux-ci figurent la pairémulation. Cette pratique de résistance ordinaire est mise en œuvre par et pour des personnes handicapées.

${ }^{2}$ Les pairémulateurs sont essentiellement des personnes présentant des incapacités motrices, physiques et/ou sensorielles.

3 Disablism : terme anglais qui signifie tout à la fois la stigmatisation (au sens de préjugés intériorisés), ségrégation (au sens de mise à l'écart spatiale) et discriminations (au sens de comportements défavorables, non-respect des droits) dirigées contre les personnes handicapées.

4 Vie Autonome: concept clef du mouvement social Independent Living Movement (ILM), "... [lequel] considère que tous les êtres humains sont interdépendants et que la notion de vie autonome ne contredit pas cette affirmation. Le terme "vie autonome" ne désigne pas l'indépendance totale vis-à-vis d'autres personnes, mais la liberté d'une personne de choisir et de contrôler sa vie et son mode de vie. " (French ILM Definitions, ENIL, 2012)
} 
leurs possibilités, leurs droits et leurs devoirs. $\|^{5}$ La pairémulation repose ainsi sur quatre conditions d'existence : la condition d'une expertise fondée sur des savoirs expérientiels de la vie autonome, la condition pédagogique relative aux modalités de la transmission, la condition éthique concernant le prérequis d'une demande formulée par le pairémulé au pairémulateur, la condition de finalité qui est une autonomisation plus aboutie. La pairémulation a ainsi pour objet la vie autonome, impliquant des objectifs de conscientisation des pairémulés concernant leurs possibilités et/ou leurs potentialités, leurs droits et également leurs devoirs.

La pairémulation est le fruit d'un triple effet de mutation dans le cadre du transfert des concepts et des pratiques du peer counseling et du peer support des USA vers la France (Gardien, 2010). Ces concepts et pratiques issus du mouvement social international pour la vie autonome (ILM) ont été rencontrés au début des années 1990 par des activistes handicapés français au cours de leurs voyages aux USA, puis dans le cadre de leurs déplacements en Europe. A partir de 1994, ces éléments furent appropriés par un collectif plus large de militants handicapés français, mais sans liens avec ce prime terreau international. De là découlent plusieurs effets de mutation. Tout d'abord, ces activistes ont introduit l'idée d'émulation au cœur de cet accompagnement par et pour les pairs. En effet, au-delà d'une pratique de conseil et/ou de soutien par les pairs, ce qui aurait correspondu à une traduction conventionnelle en français des terminologies de peer counseling ${ }^{6}$ ou de peer support ${ }^{7}, c^{\prime}$ est la référence à des notions de dépassement de soi, par l'émulation d'un pair, pour égaler ou dépasser cet autre, qui s'est progressivement imposée au fil de leurs discussions. Un deuxième effet de mutation découle du contexte de mise en œuvre. La pratique de la pairémulation a produit des écarts aux façons de faire américaines car ne se structurant pas autour d'Independent Living Centers ${ }^{8}$ et ne se fédérant pas autour d'un maillage national et inter-associatif. Un dernier effet de mutation : le terme choisi sort d'un registre de traduction littérale et n'est pas le fruit d'une simple modulation de la terminologie anglo-saxonne. II modifie en outre les représentations suscitées.

L'observation $^{9}$ des pratiques contemporaines de pairémulation a permis de les qualifier d'activité relationnelle (Demailly, 2008) pouvant endosser alternativement les registres d'action de conseil, de soutien et d'accompagnement (au sens de fournir de l'aide physique ou psychologique selon la demande et les objectifs de transformation exprimés par le destinataire, le conseiller, lui fournir de l'expertise, l'écouter, l'accompagner, le suivre), mais également le registre de l'action éducative (au sens de transformer l'habitus de l'autre de l'extérieur), de l'action thérapeutique (au sens de favoriser le retour à un état de nonsouffrance somatique ou psychique) voire de l'action persuasive (au sens de produire des modifications dans les conduites et les représentations d'autrui et de créer des désirs). Le registre d'action de l'accompagnement est très certainement celui qui correspond le plus aux discours sur leurs pratiques tenus par les pairs praticiens, celui auquel s'identifient et

\footnotetext{
${ }^{5}$ Actes des premières journées sur la Vie Autonome organisées à Paris en novembre 1994.

${ }^{6}$ Peer counseling : terminologie anglaise usuellement traduite par l'expression « conseil par les pairs ".

${ }^{7}$ Peer support : terminologie anglaise usuellement traduite par l'expression « soutien par les pairs ».

${ }^{8}$ Independent Living Centers : Centres pour la Vie Autonome.

${ }^{9}$ Une enquête ethnographique relative aux pair-accompagnements a débuté en 2010 et se poursuit encore à ce jour. Elle se déroule auprès d'une pluralité d'individus, de collectifs et d'associations, sur différents sites en territoire français. Sur certains de ces sites est pratiquée la pairémulation.
} 
vers lequel tendent nombre de pairémulateurs. La pratique elle-même est cependant plus complexe que cet idéal endossé et prôné.

\section{Vers une professionnalisation de la pairémulation}

A partir de 2002, plusieurs actions ont été menées par deux leaders pour impulser un procès de professionnalisation des activités de pairémulation, jusqu'alors essentiellement bénévoles et militantes. Des premières assises de la pairémulation ont été organisées dans la ville de Caen et ont permis de réunir les différents acteurs dispersés sur le territoire national. Ces prémisses à la constitution d'un réseau se sont poursuivies par plusieurs rencontres nationales organisées à Paris. Cela a eu pour effet de permettre aux acteurs de consolider leurs échanges en termes d'actions et de débats, de jeter les fondations d'un acteur collectif en devenir. Néanmoins la mise en œuvre d'une action concertée au niveau national entre l'ensemble des collectifs et des individus reste difficile à ce jour.

Par ailleurs, plusieurs actions de formation des pairémulateurs se sont égrenées tant dans le temps que dans l'espace (St Nizier-Le-Moucherotte, Paris, Caen, Strasbourg, entre 1998 et 2014). Toujours conçues sur un format court (entre $20 \mathrm{~h}$ et une semaine entière), elles se déroulent généralement dans une même unité spatiale dans la perspective de créer une dynamique de groupe et de susciter des échanges et des solidarités dans les temps informels. Ces formations abordent des contenus historiques et idéologique référés au mouvement pour la vie autonome, au militantisme par et pour les personnes en situation de handicap, et déclinent une présentation normative de la pairémulation (Cf. : la charte de la Pairémulation $)^{10}$. Des contenus relatifs à l'écoute d'autrui et à une posture d'accompagnement constituent la seconde partie des formations dispensées.

L'acteur collectif a également entrepris d'élaborer un référentiel d'activités et de compétences de la pairémulation. L'AFPA a d'ailleurs contribué à ce travail (2006), ainsi qu'un cabinet privé de consulting qui a conduit l'étude. Plusieurs versions ont déjà vues le jour. Le référentiel actuellement stabilisé est en attente d'être validé par le réseau des pairémulateurs, mais également par des acteurs institutionnels. Aujourd'hui, le projet consiste en l'élaboration de référentiels de formation et de certification, dans la perspective d'une demande de reconnaissance à termes d'un certificat ou d'un diplôme de pairémulateur au RNCP ${ }^{11}$.

Enfin, cet acteur collectif développe quelques activités de promotion de la pairémulation auprès des politiques, des têtes de réseau dans les champs sanitaire et médico-social, notamment auprès des centres de rééducation et de réadaptation fonctionnelles, d'institutions telles la CNSA ou la sécurité sociale, de fondations privées. Un argumentaire riche et dense en faveur de la pairémulation a été construit au fil des années et des expériences de lobbying.

Pour toutes ces raisons au moins, il est donc possible d'affirmer que la pairémulation est une activité en cours de professionnalisation. Toutefois, cette entreprise est loin d'avoir aboutie : la plupart des pairémulateurs pratiquent cette activité de manière informelle, souvent de manière irrégulière. Pour la plupart, ils ne perçoivent pas de rémunération de leur

\footnotetext{
${ }^{10}$ Charte de la Pairémulation ${ }^{\odot}$, adoptée en 2003, révisée en 2009 : http://gfph.dpieurope.org/PublicationECLAS Def/EthiqueDeontologie/ChartePairs2009Def.pdf

${ }^{11}$ RNCP : Registre National des Certifications Professionnelles
} 
intervention. L'acteur collectif est encore faible au sens où la solidarité interne et la capacité à s'organiser collectivement sont peu développées. Les reconnaissances sociales, institutionnelles et administratives de la pairémulation ne sont pas importantes, malgré un certain intérêt et un relatif soutien qui s'exprime par l'attribution ponctuelle de fonds (CRAM, CNSA, Régions, etc.). Les formations ne sont actuellement pas certifiantes, qualifiantes ou diplômantes. La spécificité revendiquée de cette pratique est néanmoins bien perçue : la transmission de savoirs expérientiels de pair à pair, supposant l'expérience du handicap et de la vie autonome.

Cette volonté même de professionnalisation suscite un fort débat au sein du réseau des pairémulateurs. Cet objectif est loin d'être consensuel. Ce qui interroge et suscite des tensions ne concerne pas la formation du pairémulateur. Ce point remporte l'adhésion générale, même si nombre de pairémulateurs exercent sans avoir été formés. Par contre, sortir d'une pratique informelle ou encore du bénévolat, concevoir une valeur monétaire aux accompagnements des pairémulateurs, collaborer avec des professionnels des champs sanitaire et médico-social, travailler avec ou dans des institutions ou encore créer un diplôme, autant de point d'achoppements structurant le débat entre militants autour de la professionnalisation de la pairémulation.

\section{Des vertus et de l'art de la relation « naturelle »}

La première ligne de tension structurant les débats entre pairémulateurs concerne donc l'opportunité de sortir de l'informel les pratiques du plus grand nombre d'entre eux. En effet, si quelques pairémulateurs sont reconnus comme tels, rémunérés (statut libéral ou salarié) pour leurs services dans un cadre associatif ou institutionnel, la plus grande part des pairémulateurs exercent de façon informelle et bénévole, au fil des rencontres, en face-àface, via Internet, etc. La pairémulation est une activité rarement désignée pour elle-même. Aussi peut-elle ne pas être connue de ceux-là même qui pourtant en bénéficient. Cette relation de soutien entre pairs se déroule bien souvent sans que sa distinction et sa nomination ne soient nécessaires. Elle participe de l'empan des relations interpersonnelles possibles entre pairs. Cette qualification de "pairémulation " n'est donc pas corollaire à sa mise en œuvre. La formalisation et la publicisation de son exercice dépendent essentiellement des pairémulateurs eux-mêmes.

« On ne va pas se pointer devant une personne qui vient d'être handicapée et qui n'a jamais entendu parler de pairémulation, ou qui est en recherche d'autonomie mais qui ne sait pas comment faire, on ne va pas lui dire : "Je suis pairémulateur, on va faire de la Pairémulation ! » " (Pairémulateur, 1 an d'ancienneté, interview du 5 juillet 2011)

Plus avant, certains pairémulateurs revendiquent de ne pas sortir des modalités informelles de leur pratique. Selon eux, formaliser la pairémulation implique de rendre des comptes aux institutions, aux professionnels, aux bénéficiaires ainsi qu'à leurs pairs. Cela suppose également de justifier de son activité, de ses résultats, de sa valeur monétaire, de son éthique, de ses effets, de ses modalités pratiques, etc. Formaliser revient donc à publiciser une identité sociale et à l'endosser dans le cadre de relations de pairémulation. Or c'est précisément sur ce premier point que s'exerce la critique : le processus même d'être présent à l'autre en tant que pairémulateur, ne modifie-t-il pas les effets de la pairémulation sur le 
pairémulé ? Ne risque-t-il pas de dévoyer la pairémulation? D’en transformer la dynamique?

« Est-ce que la professionnalisation ne risque pas de changer la relation entre le pairémulateur et le pairémulé ? Car quand je fais des confidences à quelqu'un qui a déjà vécu des choses très importantes et longues, avec qui je peux partager des choses intimes... Si je passe ensuite à une relation avec un professionnel, je n'aurais peut-être pas le même niveau d'attente. Je ne dirais peutêtre plus la même chose... Est-ce que le fait de professionnaliser ne risque pas de changer la relation ? " (Pairémulateur, vélotypie des Premières assises nationales de la pairémulation, ${ }^{\text {er }}$ avril 2011, p.17)

L'inscription de la pairémulation au cœur des relations interpersonnelles ordinaires, entre pairs, est un enjeu majeur. II s'agit pour ces pairémulateurs de préserver une relation "naturelle" en quelque sorte, soumise à aucune autre obligation ou entrave que l'engagement réciproque et volontaire dans une relation humaine. Par conséquent, les rapports d'expertise sont congédiés. Pareillement, ces pairémulateurs soutiennent une posture sans cette " distance à l'autre " qui caractérisent les professionnels. Ils refusent de s'inscrire dans des rapports surplombants et prônent des relations égalitaires. Pour ces pairémulateurs, leur pratique consiste avant tout en une façon d'être, une posture relationnelle avec ses pairs, plutôt qu'en un métier ou une expertise.

« - J'ai bien l'impression qu'être pairémulateur devient plus un métier qu'une façon d'être... Or c'est quelque chose que beaucoup de gens font naturellement. Là, on le fait passer pour un métier. Est-ce qu'être pairémulateur sera maintenant un métier?" (Pairémulatrice, vélotypie des Premières assises nationales de la pairémulation, $1^{\mathrm{er}}$ avril 2011, p.33)

Globalement, l'ensemble des pairémulateurs s'inquiète de ressembler à des professionnels. La plupart redoutent de devenir comme eux sous l'effet d'une professionnalisation. Ils craignent véritablement d'être considérés comme des professionnels par les pairémulés et par suite de ne plus pouvoir œuvrer. Aussi, se professionnaliser est envisagé comme prendre le risque de changer la relation, et surtout d'en altérer la nature et les effets.

«Cela dit, on est sur un fil : je ne veux pas endosser la blouse blanche. Je veux garder ce côté informel. Je ne veux pas prendre de notes pendant la discussion [avec le pairémulé], je note après l'échange [...] II va être difficile de professionnaliser en gardant ce côté informel. " (Pairémulateur, 24 ans d'ancienneté, interview du 22 avril 2013)

\section{Libertés du bénévolat et contraintes du statut professionnel}

D’autres pairémulateurs contestent l'idée de renoncer au bénévolat ${ }^{12}$ pour lui-même, qu'il soit formel ou informel. C'est à ce titre qu'ils récusent la professionnalisation. Plusieurs raisons à cela.

\footnotetext{
${ }^{12}$ Le bénévolat est ici considéré dans son acception extensive et ne se résume au bénévolat organisé, à savoir : "Le bénévolat est un don de temps, c'est-à-dire une activité volontaire, sans rémunération, et orientée vers autrui. Cette dernière condition est essentielle pour que l'on puisse parler de don. Néanmoins, tel qu'il est ainsi défini, le don de temps recouvre des pratiques fort nombreuses qui ne sont pas toutes inscrites au registre du bénévolat. L'accord prévaut généralement pour exclure les sphères professionnelles et domestiques. » (Prouteau, $2001: 20$ )
} 
Tout d'abord, pour eux, être bénévole permet de ne pas avoir à rendre de comptes de façon systématique à une institution ou à une hiérarchie. Le bénévolat est donc conçu comme une marge de liberté, comme un champ des possibles moins corseté que celui des professionnels. L'institution est perçue avant tout comme contraignante.

Dans cette perspective, le bénévolat permet également d'affirmer des positions fortes, si nécessaires, sans craindre d'éventuelles sanctions qui mettraient le pairémulateur dans l'obligation de choisir entre se soumettre ou perdre son salaire. Les pairémulateurs tenants de cette position s'inquiètent de situations de pairémulés dont les droits ne sont pas respectés par l'institution qui les accueillent. Ainsi, ils envisagent la possibilité de désaccords ou de tensions vives entre le pairémulateur, l'institution dans son ensemble et/ou certains professionnels. Le bénévolat devient alors un statut opportun car il conforte la possibilité de rester sur des positions éthiques fortes.

« C'est la première fois de ma vie que je me suis fait licencié ! Licencié... Enfin j'étais bénévole donc c'était difficile, mais j'ai été remercié ! [...] On nous a dit : « ce que vous proposez est trop perturbant pour l'institution. " Finalement... Cela n'a pas été dit comme ça, mais c'était le message que nous avons reçu ! Heu... C'est trop d'insécurité... C'est trop... Trop perturbant pour l'institution ! Pour le fait institutionnel...» (Pairémulateur, 20 ans d'ancienneté, interview du 22 septembre 2010)

Par ailleurs, et si besoin, le bénévolat permet de se désengager de l'activité et/ou de son contexte d'exercice sans les contraintes de la relation contractuelle. C'est à ce titre que le bénévolat est privilégié. II peut être facilement réinvesti dans d'autres contextes, avec ou sans encadrement. Aussi, le bénévolat est revendiqué pour l'engagement souple et sur mesure qu'il permet, pour la liberté qu'il procure, pour l'éthique qu'il permet de tenir.

Un autre avantage du bénévolat perçu par quelques pairémulateurs est la possibilité de ne pas accepter ou subir la normativité d'une équipe de professionnels. Le bénévolat contraindrait moins le pairémulateur dans ses actions. Il garderait davantage la possibilité de ne pas appliquer des principes collectifs auxquels il n'adhère point. Le bénévole pourrait davantage revendiquer de penser et d'agir en son nom, par lui-même, car il peut toujours se saisir d'une opportunité pour aller pratiquer bénévolement ailleurs, sans que cela remette en cause même temporairement ses capacités financières et celles de ses proches. Ainsi, le bénévolat peut être perçu et préféré en raison de la marge de manœuvre qu'il procure.

«Moi c'est un peu délicat car lorsque j'interviens dans une structure de rééducation... C'est tellement dur de rentrer dans ces structures! Je suis toujours un peu en porte à faux car je reste persuadé que les médecins, thérapeutes n'ont pas... Je les vois faire certaines pratiques et je ne suis pas d'accord du tout. Ça m'est difficile de leur dire qu'ils sont à côté de la plaque. "Faite plutôt ce que je vous conseille ": ce n'est pas évident! C'est la difficulté de travailler dans ces structures. J'espère qu'il y aura d'autres tentatives de ne pas se mettre à dos le personnel tout en essayant de faire profiter la personne [handicapée] d'autres expériences. " (Pairémulateur, 24 ans d'ancienneté, interview du 21 août 2012)

Un autre argument en faveur du bénévolat est la volonté de ne pas créer de scission ou de ligne de partage dans le réseau des pairémulateurs. Professionnaliser suppose d'établir des attentes et des exigences minimales (notamment en termes de niveau et de prérequis), les compétences à acquérir, de mettre en œuvre une sélection à l'issue de la formation. Aussi, s'orienter vers la professionnalisation de la pratique de pairémulation aura pour effet de 
distinguer les " bons " pairémulateurs des autres, ceux qui seront alors leur pâle copie. Pour cette raison la professionnalisation est vécue par certains comme une mise en concurrence des légitimités entre pairs, et par suite comme un risque de clivage en sein du réseau.

"Pour compléter, je n'osais pas le dire... Je trouve dommage de se caler sur un niveau bac, même si je comprends... Pour moi, la Pairémulation est une posture plus qu'une compétence. Je connais des gens avec bac+ 9 qui seraient incapables d'être des pairémulateurs. Et je connais des gens qui ont bac- 9 et qui seraient d'excellents pairémulateurs. Donc, ça me pose souci " (pairémulateur, vélotypie des Premières assises nationales de la pairémulation, $1^{\mathrm{er}}$ avril 2011, p.38)

Cette crainte de la professionnalisation est partagée par plus d'un. D'ailleurs l'idée d'une formation diplômante interroge: qu'en sera-t-il des pairémulateurs qui n'ont pas le niveau scolaire préalable ? Rempliront ils néanmoins les prérequis pour entrer en formation ? Quid de la sélection à l'entrée et à la sortie de la formation pour les pairémulateurs déjà en activité ? Quid de ceux qui échouent aux examens ? Une VAE sera-t-elle proposée ?

Autrement dit, si les praticiens de longue date n'ont pas le niveau requis, pourront-ils continuer à s'auto-qualifier de pairémulateurs ou bien leur activité va-t-elle retomber dans l'ordinaire des relations quotidiennes et eux se retrouver disqualifiés ?

«Peut-être manque-t-il quelque chose ? Puisque ça sera un nouveau métier, une fois la fiche métier validée... II manque peut-être la validation des acquis de l'expérience. Dans la salle, vous avez déjà un potentiel de personnes... " (Pairémulateur, vélotypie des Premières assises nationales de la pairémulation, $1^{\mathrm{er}}$ avril 2011, p.31)

D'autres s'enquièrent de leurs légitimités à se professionnaliser. Peuvent-ils vraiment revendiquer un statut professionnel pour cette activité ? Jusqu'où et à partir de quand peuton se prendre à ce point-là au sérieux ? D'autres encore précisent les risques encourus par l'élaboration d'une normativité par la sélection au diplôme, ou encore par le travail en équipe entre pairs ou avec d'autres professionnels.

\section{Valeurs de la pairémulation : reconnaissance et rémunération}

D'autres questions se posent encore, et notamment celle de la valeur de leurs interventions. Les pairémulateurs font consensus autour de l'idée qu'un fort préjugé existe autour des situations de handicap, faisant de toute activité un élan de charité ou un don de soi. Le bénévolat serait ainsi une évidence pour les institutionnels comme pour les professionnels ou les proches. Les personnes handicapées auraient beaucoup de temps disponible à offrir, du fait même de leurs incapacités.

« On est face à un travers de cette société. On pense que, dès qu'il s'agit de handicap, ça devrait être gratuit et spontané. [...] C'est un état d'esprit très ancré, dont il est difficile de se défaire. Et les personnes handicapées sont entrées dans ce système. Elles n'osent pas dire : « je suis professionnel, ça coûte tant ». II faut franchir le pas. II y en a qui y arrivent, d'autres moins. » (Pairémulateur, vélotypie des Premières assises nationales de la pairémulation, $1^{\text {er }}$ avril 2011, p.24)

Cependant, les pairémulateurs s'interrogent de concert : une personne handicapée ne peutelle être compétente? Qui plus est, ne peut-elle être compétente du fait même de son expérience du handicap ? Ses savoirs expérientiels n'ont-ils pas une certaine valeur, voire 
une valeur certaine ? C'est la perspective défendue par tous les pairémulateurs, déjugeant l'évidence du bénévolat, exigeant qu'il procède d'un choix et non d'une contrainte dévaluative exercée arbitrairement de l'extérieur par des valides.

Aussi, très logiquement, une minorité de pairémulateurs exige-t-elle une rémunération en échange de leurs services. Pour ces derniers, il est normal de demander une rémunération pour ce qu'ils considèrent comme un travail13. "Tout travail mérite salaire ", reprennent-ils alors en cœur. L'argent a une fonction de reconnaissance et de valorisation de l'activité à leurs yeux. La rémunération est également une preuve de leur traitement à égalité avec les valides et donc l'emblème de leur dignité. Dans cette optique, cette minorité souhaite d'ailleurs s'accorder sur une valeur monétaire de la prestation fournie.

Plus généralement, les pairémulateurs sont partagés quand aux conséquences de ces gains sur leur sécurité financière et donc sur leur avenir. Certains mettent en avant la nécessité d'une rémunération pour assurer leur subsistance, tandis que d'autres ou les mêmes jaugent le risque de perdre leur emploi et de ne pouvoir retrouver la sécurité de l'Allocation Adulte Handicapé dans l'immédiat.

"Après, il va y avoir un problème de fond. Ça fait au moins cinq ou six ans qu'on en parle... Si j'ai un métier, est-ce que je ne risque pas de perdre mon AAH ? Si oui, c'est le retour à la situation antérieure en cas d'échec sur le marché du travail. Si je vois que ça ne marche pas, je veux pouvoir arrêter et ne pas être obligé d'attendre un ou deux ans avant d'avoir à nouveau des droits auprès de la MDPH. " (Pairémulateur, vélotypie des Premières assises nationales de la pairémulation, $1^{\mathrm{er}}$ avril 2011, p.34)

Enfin, quelques pairémulateurs rappellent l'importance de fonds substantiels pour assurer la qualité de leurs interventions, ne serait-ce que pour financer d'éventuels frais de structure. Ces perspectives doivent se comprendre à l'horizon de projets de structuration des activités de pairémulation en services d'accompagnement à la vie autonome. L'argumentaire déployé alors pour convaincre les politiques, collectivités territoriales et institutions diverses de subventionner ces activités met en avant l'utilité publique de la pairémulation, également le fait que son déploiement à l'échelle permettrait de combattre le chômage massif et endémique des personnes handicapées en France.

\section{Collaborer avec des professionnels, travailler en institution ?}

Ceci étant dit, la discussion la plus vive est certainement celle se rapportant à la collaboration avec les professionnels des champs du sanitaire et du médico-social. Faut-il ou non travailler en lien avec ces professionnels et ces institutions ? Le débat est endémique, sans jamais être tranché. Plusieurs registres d'argumentation sont déployés en réponse à cette question.

De manière quasi unanime, les pairémulateurs mettent en avant un paradoxe qui nuit aux collaborations ou partenariats avec des professionnels des secteurs sanitaire et médico-

\footnotetext{
${ }^{13}$ Les pairémulateurs s'inscrivent dans une conception restrictive du "travail ». Cette catégorie est associée par eux à celles d'emploi, de contrepartie monétaire et d'inscription dans la sphère marchande. Le travail serait une activité économique d'abord.
} 
social. Le pairémulateur serait identifié comme une personne handicapée, à comprendre au sens de déficitaire. Par suite, le pairémulateur serait perçu et traité comme une personne bénéficiaire $d$ 'un accompagnement. Autrement dit, le pairémulateur susciterait malgré lui des représentations en termes d'incapacités, d'incompétences, voire évoquerait un besoin de protection et d'accompagnement. Paradoxe donc, car le propre du pairémulateur est justement, a contrario, sa capacité d'autonomie, voire son indépendance ${ }^{14}$. II semble donc qu'il soit difficile d'endosser un autre rôle que celui de bénéficiaire pour un pairémulateur face à des professionnels aguerris, autonomie ou pas. Aussi, ce constat interroge sur la possibilité réelle pour cette activité d'être reconnue à légitimité égale, bien qu'elle soit pourtant spécifiquement tendue vers l'autonomie et portée par des individus détenteurs d'une expérience de la vie autonome.

" J'aurais tendance à dire qu'être pair ne suffit pas à être légitime. Il faut se rendre à l'évidence! Pour les professionnels ? Non ça ne leur suffit pas! Y'a toujours quand même la blouse blanche qui fait autorité. Dans le milieu médico-social quand tu n'es pas identifié [...] Je pense qu'ils aiment bien avoir des repères précis et nous on descend un peu d'une autre planète. " (Pairémulateur, 4 ans d'ancienneté, interview du 21 août 2012)

«Comment peut-on faire en sorte pour que [dans les représentations] ce ne soit plus forcément la personne handicapée qui ait besoin d'assistance? Qui soit bénévole? Bénéficiaire ? " (Pairémulatrice, 1 an d'ancienneté, interview du $1^{\text {er }}$ juin 2012)

Un autre souci des pairémulateurs est l'absolue nécessité, sauf à mettre en péril leurs accompagnements, de ne pas être identifié en tant que " professionnel " par les pairémulés, tout en étant possiblement membres d'une équipe soignante, sociale et/ou socio-éducative. Afin de préserver un cadre spécifique et nécessaire au bon déroulement de l'échange entre pairémulateur et pairémulé, ces praticiens ont établi un certain nombre de normes, au nombre desquelles: ne pas porter de blouse blanche, avoir une souplesse horaire lors de la rencontre, fonder la relation sur l'échange réciproque d'expériences, etc. Or, comment arriver à faire valoir la spécificité des modalités relationnelles de la pairémulation auprès des professionnels? Autrement dit est-il possible de faire partie d'une équipe tout en se distinguant franchement des normes de la relation professionnelle à l'usager, que ce soit en termes de maintien de la "bonne distance à l'usager ", de non engagement affectif ou personnel, etc. ?

" Vous allez perturber un certain nombre d'équipes à ne pas enfiler des blouses blanches. Donc ça va être ressenti comme une agression... " (Pairémulateur, vélotypie des Premières assises nationales de la pairémulation, $1^{\text {er }}$ avril 2011, p.34)

Est-il envisageable que les pairémulateurs puissent refuser de participer aux rapports de pouvoir établis entre professionnels et usagers ? En effet, pour les pairémulateurs, c'est d'abord la possibilité d'un échange interpersonnel ordinaire, de pair à pair, dans la

\footnotetext{
${ }^{14}$ Indépendance : ce terme est à comprendre, dans ce contexte très spécifique, comme l'ensemble des possibilités de «faire par soi-même » qu'inventent les pairémulateurs dans les situations concrètes où le corps différent n'apparait pas spontanément efficient. Ces nouveaux usages du corps et de son environnement font partie de la panoplie des savoirs expérientiels développée par les pairémulateurs. L'indépendance correspond donc à des possibilités pratiques de faire quelque chose, tandis que l'autonomie fait référence aux possibilités plus intellectuelles de choisir, décider, s'organiser, etc.
} 
réciprocité et sans asymétrie, qui fonde leur pratique. La relation porte couramment sur des pans intimes de l'existence, très liés à l'expérience même du handicap. Cette intimité réciproque suppose la confidentialité (a minima) comme condition d'existence. Mais, dans quelle mesure, s'inscrire dans une équipe de professionnels n'est-ce pas devoir endosser les normes relationnelles qui y sont en vigueur, à savoir : le partage des informations recueillies auprès de la personne handicapée ?

Or, du point de vue des pairémulateurs, la confiance réciproque entre pairémulé et pairémulateur est à ce point nécessaire à la qualité de l'accompagnement, que les pairémulateurs jugent essentiel d'obtenir la possibilité d'un "secret" spécifique au pairémulateur, qui les dédouanerait du partage des informations recueillies à l'équipe. En effet, respecter la parole du pairémulé c'est également respecter son adresse. Si ces mots ont été adressés au pairémulateur, $c^{\prime}$ est dans le cadre de cette relation très spécifique suscitée par la commune expérience du handicap. Aussi, les pairémulateurs s'interrogent: n'est-ce pas la possibilité de s'inscrire dans un " secret partagé » qui participe à fonder la place de membre d'une équipe ? Cette condition d'un droit au secret pour le pairémulateur n'est-elle pas rédhibitoire à tout véritable travail en équipe ?

« Parce que moi, lorsque j'ai travaillé en centre de rééducation à $X$, la difficulté que j'ai rencontré dans la relation aux professionnels c'est qu'ils voulaient tout savoir... II faudrait pouvoir établir un droit au secret... » (Pairémulateur, 15 ans d'ancienneté, interview du 24 juin 2013)

En outre, les pairémulateurs s'inquiètent des réticences et des peurs des professionnels visà-vis de leurs pratiques et de leurs apports spécifiques. Dans leur perspective, le pair par sa capacité à partager son expérience du handicap avec la personne handicapée, cette pratique peut être un enrichissement dans le panel des accompagnements et soins apportés. Aussi, une complémentarité des interventions leur semble envisageable. Pour autant, les expériences de pairémulation en institution montrent une tendance à la mise en concurrence ou encore à la rivalité des professionnels vis-à-vis de ce nouveau mode d'accompagnement. Comment faire pour apaiser les résistances de ces professionnels? Pour apaiser leurs craintes ? Pour limiter les rapports de pouvoir trop violents, ou encore une volonté d'emprise sur ces pratiques émergentes dans le champ institutionnel ? Faut-il accompagner ces professionnels dans cette introduction d'une nouvelle fonction professionnelle, pour les aider à faire face à leurs inquiétudes et aux changements suscités ? Autant de questions posées mais dont les réponses ont du mal à s'élaborer. Quelques expériences en institution se poursuivent au jour d'aujourd'hui. Lorsqu'elles sont portées favorablement par les professionnels, de nombreux patients ou résidents sont orientés vers le pairémulateur. La mise en contact en est ainsi facilitée.

« [...] car les professionnels de santé ne vont pas supporter : " c'est qui ces gens sans blouse blanche qui viennent jouer dans notre cour de récréation ? " (Pairémulateur, 15 ans d'ancienneté, interview du 22 août 2012)

Enfin, une question récurrente vient profondément laminer une possible alliance entre pairémulateurs et professionnels des secteurs sanitaire et médico-social. En effet, l'autonomie que tous appellent de leurs vœux semble un objectif partagé sur lequel s'appuyer pour travailler ensemble. Mais le doute plane du côté de pairémulateurs sur le fait que les professionnels aspirent et œuvrent véritablement à l'autonomie des personnes handicapées. En effet, leurs pratiques s'inscrivent beaucoup trop dans le projet de 
développement d'une indépendance corporelle et fonctionnelle maximale, dans l'inscription des personnes handicapées dans une vie en institution, et trop peu dans le déploiement d'une capacité à l'autodétermination.

"Quand on voit le nombre d'orientations qui sont encore faites vers les foyers de vie, les maisons d'accueil où les tarifs sont prohibitifs, quand on voit qu'on développe plutôt ces solutions plutôt que de l'accompagnement à l'autonomie... C'est flagrant en France! On voit encore beaucoup de gens qui pourraient être orientés vers de l'autonomie qui ne le sont pas. Ce qui n'est pas le cas dans les pays nordiques. " (Pairémulateur, 15 ans d'ancienneté, 22 août 2012)

En outre, très couramment, les pairémulateurs constatent une divergence sérieuse en matière d'estimation du potentiel des personnes handicapées. Selon eux, les professionnels focalisent sur les incapacités et la réduction du champ des possibles, et ne perçoivent pas toujours, ou alors mal, le potentiel à développer ou encore les capacités à faire autrement. Partant de là, les pairémulateurs s'interrogent sur l'écoute qu'on voudra bien accorder à leurs perspectives et à leurs savoirs expérientiels, également sur la crédibilité et la légitimité que les professionnels pourraient attribuer à leurs pratiques.

\section{Conclusion}

Si la professionnalisation de la pairémulation a tout d'abord essentiellement consisté en des activités de formation et la construction progressive d'un acteur collectif, un virage important a été pris au début des années 2000 avec le projet d'élaborer un référentiel d'activités et de compétences. Cette initiative a tout d'abord été portée au sein du collectif du Calvados. Un premier référentiel a alors été produit en 2006. Puis ce nouvel outil a été retravaillé dans le cadre de rencontres nationales de pairémulateurs, lesquelles ont permis une adhésion et une appropriation de l'outil par un noyau dur de l'acteur collectif.

Pour autant, Le consensus autour de ce référentiel de capacités et de compétences n'est pas acquis. Dans la nébuleuse des individus et collectifs se revendiquant de la pairémulation, les résistances à l'idée de pousser plus avant la professionnalisation (création de référentiels formation et certification ainsi que leur mise en œuvre) est importante. Plusieurs niveaux d'argumentations fondent ces positions divergentes: la volonté de conserver une pratique informelle pour les effets et résultats qu'elle permet, le choix de demeurer dans un statut bénévole pour les libertés que cela procure. En outre, le risque en termes de chômage et de perte de certaines allocations qu'implique la demande d'une rémunération, ainsi que les résistances courantes des professionnels et du milieu institutionnel à l'introduction de cette pratique dans les établissements sanitaires et médico-sociaux forgent un contexte défavorable aux yeux de nombre de pairémulateurs.

La pratique de la pairémulation se décline donc concrètement selon une variété de modalités et de statuts : du bénévolat informel au statut salarié ou libéral, en passant par le bénévolat formel. Cette pluralité peut également être analysée en termes de positionnements individuels relatifs à une demande de reconnaissance, dans le cadre plus large d'une construction sociale et politique de ladite reconnaissance portée par l'acteur collectif, et progressivement par des acteurs institutionnels ${ }^{15}$.

\footnotetext{
${ }^{15}$ Une mission a été confiée à madame Marie-Sophie Desaulle le 2 décembre 2014 par le Secrétariat chargé des Personnes handicapées et de la lutte contre l'exclusion dont l'un des quatre axes de travail est intitulé
} 
Cette hypothèse est confortée par les distinctions opérées entre bénévolat formel et informel par des chercheurs spécialistes de ce champ (Duschesne, 1989 ; Prouteau, 2001). Le bénévolat informel y est situé statistiquement comme plus répandu que le bénévolat formel, plus féminin, inscrit dans des relations de réciprocité. La reconnaissance par autrui concerne alors le réseau de proximité. Le bénévolat formel, quant à lui, plus masculin, apparaît à l'analyse comme davantage relié à un besoin de réalisation personnelle, à un intérêt pour un élargissement des réseaux de sociabilité au-delà du cadre de la famille ou du voisinage, à des attentes de reconnaissance et de gratifications en retour par les associations ou organisations qui bénéficient de ce don de temps et de compétences. La rémunération et l'inscription dans un statut salarié ou libéral peuvent également se comprendre comme une demande de reconnaissance à un niveau plus sociétal.

Ces trois positions observables chez les pairémulateurs, peuvent trouver leur modèle d'analyse dans la typologie des formes de reconnaissance proposée par Axel Honneth (2000). Le bénévolat informel pourrait être revisité à l'aune du concept de "sollicitude personnelle ", concept afférant à la sphère des relations primaires, des soutiens affectifs, instaurant la confiance en soi. Le concept $d$ ' " estime sociale " désignant la reconnaissance des capacités et qualités propres de l'individu, de son utilité sociale, permettrait un approfondissement de la connaissance des dynamiques sous-tendant le bénévolat formel et les statuts de salarié ou de libéral.

\section{Bibliographie}

Demailly, L. (2008). Politiques de la relation: approche sociologique des métiers et des activités professionnelles relationnelles. Villeneuve d'Ascq: Presses universitaires du Septentrion.

Duschene, D. (1989). Donner sans compter : les bénévoles au Canada. Ottawa : Statistiques Canada.

Gardien, E. (2010). La pairémulation dans le champ du handicap : Histoires, pratiques et débats en France. Rhizome, 40, 2-3.

Honneth, A. (2000). La lutte pour la reconnaissance. Paris : éd. Du Cerf

Prouteau, L. (2001). Les figures du bénévolat. Recherches et prévisions, 63, 19-32.

«Création d’une dynamique d’accompagnement et de soutien par les pairs : personnes concernées et famille pilote défenseur des droits? " 